\title{
Sinus Bradycardia by ECG Finding
}

National Cancer Institute

\section{Source}

National Cancer Institute. Sinus Bradycardia by ECG Finding. NCI Thesaurus. Code

C111097.

An electrocardiographic finding of abnormally slow heart rate with its orig in in the sinus node. Thresholds for different age, gender, and patient populations exist. (CDISC) 